\title{
ALGUNOS APUNTES SOBRE DESACUERDOS TEÓRICOS INTERDISCIPLINARIOS ENTRE EL DERECHO Y LA ECONOMÍA
}

\author{
SOME NOTES ON INTERDISCIPLINARY THEORETICAL \\ DISAGREEMENTS BETWEEN LAW AND ECONOMICS
}

\author{
FABRIZIO EsPOSITO*
}

\begin{abstract}
Resumen:
El punto de partida de este ensayo es la pregunta “¿Bajo qué condiciones se justifica que quienes ejercen derecho ignoren el punto de vista económico?". Esta pregunta nos lleva a una investigación sobre la relación entre los desacuerdos que los economistas tienen con el derecho y los desacuerdos teóricos. Este ensayo hace dos afirmaciones principales. En primer lugar, los desacuerdos que los economistas tienen con el derecho pueden originar un tipo particular de desacuerdo teórico: un desacuerdo teórico interdisciplinario. Los desacuerdos teóricos interdisciplinarios presuponen la solución de un problema de traducción desde la economía al derecho. El problema de la traducción se resuelve cuando una proposición de la economía se convierte en parte de la justificación externa de una norma jurídica. Tiene sentido utilizar la expresión 'traducción interdisciplinaria' (pero también transportación, transferencia) porque el significado se traslada de una práctica a otra. En segundo lugar, las diversas posiciones respecto de la relación entre el derecho y la moral son también un problema de traducción interdisciplinaria - en este caso desde la moral al derecho. A la luz de esta comprensión, el ensayo concluye con la esperanza de que los filósofos del derecho y los teóricos del derecho se interesen más por la relación entre el derecho y la economía.
\end{abstract}

Palabras clave: Desacuerdo teórico interdisciplinario; Problema de traducción interdisciplinaria; Justificación externa; Derecho y Economía; Derecho y moral

* U.C. de Lovaina, Bélgica (fabrizio.esposito@eui.eu). Una versión previa de este artículo fue publicada en italiano en Alcune Note sui Disaccordi Teorico-Interdisciplinari del Diritto con l'Economia, "Rivista Internazionale di Filosofia del Diritto", S5 (1), 2017, pp. 91-117. El autor agradece al consejo editorial de la revista el permiso para volver a publicar el artículo. En comparación con el original, hay actualizaciones mínimas en las notas al pie. Artículo recibido el 11 de mayo de 2020, y aceptado para su publicación el 16 de julio de 2020. Traducción del inglés de Ernesto Riffo Elgueta. 


\begin{abstract}
:
The starting point of this essay is the question "Under what conditions is the legal practitioner justified in ignoring the economic point of view?". This question leads to an inquiry of the relation between the disagreements economists have with the law and theoretical disagreements. The essay makes two main claims. First, the disagreements economists have with the law can originate a particular kind of theoretical disagreement - an interdisciplinary theoretical disagreement. Interdisciplinary theoretical disagreements pre- suppose the solution of a translation problem from economics into law. The translation problem is solved when a proposition of economics becomes part of the external justification of a legal norm. It makes sense to use the expression «interdisciplinary translation» because meaning is moved from one practice to another. Second, the various positions with regard to the relation between law and morality are also a problem of interdisciplinary translation - this time from morality to law. In light of this insight, the essay concludes with the hope of more interest by philosophers of law and legal theorists for the relation between law and economics.
\end{abstract}

Keywords: Interdisciplinary Theoretical Disagreement; Interdisciplinary

Translation Problem; External Justification; Law and Economics; Law and Morality

\title{
I. INTRODUCCIÓN
}

Ocurre que los economistas, como todos, no están de acuerdo con lo que el derecho les exige hacer. Lo que caracteriza a los economistas y, en un sentido, los hace un poco especiales, es la unión de una notoria arrogancia intelectual ${ }^{1}$ (el así llamado "imperialismo económico")2 con una fuerte reticencia a discutir cuestiones de valor porque no son científicas. La consecuencia es un acercamiento a los estudios de derecho que suele ser unidireccional, desde la economía hacia el derecho. ${ }^{3}$

1 La mejor manifestación de esta circunstancia es quizás la pregunta “¿es usted economista?" planteada en conferencias interdisciplinarias por economistas que presentan un análisis económico del derecho sin, obviamente, "ser juristas".

2 Véase, por ejemplo, MäKi (2013), pp. 325-339 y LePenies y MALECKa (2015).

3 Este punto está en el centro de la discusión de la "economic jurisprudence" ofrecida por CAlabresi (2016). Para una discusión útil y ágil del análisis de CAlabresi, ver ChiassSONI (2016) pp. 608-615. He explorado la profundidad y el alcance de la contribución de CALABresi al análisis económico del derecho en Esposito (2017), pp. 375-406, y Esposito (2019), pp. 1-11. 
En este artículo, investigo la relación entre los desacuerdos que los economistas tienen con el derecho y el tipo de desacuerdo que Dworkin ha llamado (no muy felizmente) ${ }^{4}$ desacuerdo teórico [theoretical disagreement]. En el centro de esta investigación está la pregunta: "¿Cuáles son las circunstancias en las que el jurista está justificado en desconocer la perspectiva económica?”. Sostengo dos tesis principales. Primero, los desacuerdos de los economistas con el derecho pueden dar lugar a un tipo particular de desacuerdo teórico, al que llamo desacuerdo teórico interdisciplinario. Sin embargo, para que surja este tipo de desacuerdos, es necesario resolver un problema de traducción interdisciplinar, desde la economía hacia el derecho. Este problema puede resolverse incorporando una proposición económica en la justificación externa de una proposición jurídica. Bajo esta perspectiva, puede hablarse de traducción interdisciplinar (o incluso de transición, transporte, transferencia) porque el significado se traslada de una práctica lingüística a otra.

En segundo lugar, los puntos de vista teóricos sobre la relación entre el derecho y la moral son también un problema de traducción interdisciplinaria, esta vez desde la moral hacia el derecho. Esto me lleva a sugerir a los filósofos y teóricos - del derecho - la oportunidad de interesarse más por la relación entre el derecho y la economía. Las siguientes consideraciones son, en efecto, solo uno de los ejemplos del hecho de que el bagaje conceptual de la filosofía y la teoría del derecho representa una fuente extremadamente valiosa para el estudio de la relación entre el derecho y la economía.

El ensayo está estructurado como sigue. Comienzo presentando en la sección $\S 2$ algunos ejemplos sencillos de proposiciones interdisciplinarias, seguidos de una explicación de los desacuerdos interdisciplinarios entre la economía y el derecho. En la sección $\S 3$, ofrezco una breve descripción de la crítica dworkiniana a cómo los positivistas han explicado los desacuerdos teóricos, así como de las principales réplicas positivistas. Esto me permite distinguir dos tipos de desacuerdos interdisciplinarios: a) desacuerdos teóricos interdisciplinarios; y b) desacuerdos puramente interdisciplinarios. Los participantes en la práctica jurídica pueden tranquilamente ignorar los desacuerdos puramente interdisciplinarios. Sin embargo, esta respuesta no es satisfactoria, siempre que no se excluya la posibilidad de que un desacuerdo que parezca ser puramente interdisciplinario no pueda convertirse, después de una traducción, en un desacuerdo teórico interdisciplinario. Por tanto, debe abordarse el problema de la traducción interdisciplinaria.

4 Ratti y Dolcetti (2013), p. 305, observan en particular que esta expresión "evoca la idea de una explicación científica de un determinado fenómeno; sin embargo, el fenómeno de los desacuerdos jurídicos es el resultado de una divergencia entre doctrinas evaluativas (es decir, teorías normativas) sobre las fuentes y su significado". 
Papayannis se ha ocupado recientemente del problema de la traducción. Lo abordó en particular respecto de los análisis económicos funcionales del derecho. En la sección $\S 4$, observo que Papayannis admite la posibilidad de la traducción, pero no investiga adecuadamente el punto. Por tanto, es útil evaluar un debate entre Patterson y Craswell sobre la traducción del concepto de contratos incompletos desde la economía hacia el derecho. En este caso, la conclusión sobre la posibilidad de traducción también es positiva. Sin embargo, se pone la atención en la necesidad de avalar [warrant] (o validar) las proposiciones económicas de acuerdo con los procedimientos para ello del discurso jurídico. A la luz de esto, concluyo que la traducción interdisciplinaria concierne a la relevancia de una proposición económica para la justificación externa de una proposición de derecho.

En la sección V, procedo a defender la segunda tesis de este ensayo: el debate sobre la relación entre el derecho y la moral puede reformularse en términos de posiciones teóricas acerca de las condiciones bajo las cuales es posible traducir (o incluso transitar, transportar, transferir) una proposición desde la moral al derecho. Sobre la base de este resultado, hago dos consideraciones: $a$ ) los filósofos y teóricos del derecho pueden hacer una contribución importante al estudio de la relación entre el derecho y la economía; $b$ ) el problema de la traducción de proposiciones interdisciplinarias es un problema que merece más atención.

\section{PROPOSICIONES ECONÓMICAS INTERDISCIPLINARIAS: CUANDO LA ECONOMÍA HABLA DE DERECHO O LE HABLA AL DERECHO}

Una proposición interdisciplinaria es el contenido de un enunciado que está avalado [warranted $]^{5}$ (es considerado válido, verdadero, correcto) de acuerdo a las reglas de una práctica, pero que concierne a otra práctica, que es por tanto el objeto de la proposición interdisciplinaria. En la perspectiva de la práctica objeto, la proposición interdisciplinaria es, por tanto, una metaproposición, ya que pertenece a un metalenguaje. En la vida cotidiana usamos habitualmente una amplia gama de posiciones interdisciplinarias: "el uniforme de la Juventus es horrible" es una proposición estética que concierne al juego del fútbol; "Si te

5 Utilizo "aval" [warrant] de acuerdo con HAAck (2015) en particular pp. 19-21. De otra forma, podría utilizarse la distinción inferencialista entre compromiso [commitment] y habilitación [entitlement]. Sobre este punto, ver CANALE y Tuzet (2007), pp. 32-44 y CANALE y Tuzet (2007b), pp. 73-89. La limitación de este enfoque para los propósitos actuales es principalmente desviar la atención desde las afirmaciones y proposiciones hacia los participantes y al "scorekeeping" entre ellos. 
bañas después de comer, te duele el estómago" es una proposición médica (o de un padre aprensivo) que concierne a la actividad del baño.

Por supuesto, también pueden existir desacuerdos interdisciplinarios. En general, existe un desacuerdo cuando, en relación con algo, un aseverador A1 afirma (o implica [implies]) una proposición $P$ mientras que un aseverador A2 afirma (o implica) una proposición diferente respecto de $P{ }^{6}{ }^{6}$ Por ejemplo, alguien podría sostener que "el uniforme de la Juventus es hermoso" o que "habiendo comido ligero, no me dolerá el estómago si me baño". De esta forma, tenemos un desacuerdo con quienes creen que el uniforme de la Juventus es horrible y con quienes afirman que bañarse después de comer te enferma incluso si has comido liviano. En los desacuerdos interdisciplinarios recién vistos, hay dos proposiciones $P 1$ y $P 2$ pertenecientes a una práctica que tiene como objeto una proposición $P x$ perteneciente a una práctica diferente: $P 1(P x)$ vs $P 2(P x)$. Más sucintamente, existe un desacuerdo interdisciplinario cuando hay dos proposiciones interdisciplinarias discordantes que tienen como su objeto la misma proposición de la práctica objeto.

Otro tipo de desacuerdo interdisciplinario surge, por ejemplo, si a la pregunta "señor, ¿no cree que el nuevo uniforme de la Juventus es horrible? ¿No sería mejor cambiarlo?", el técnico respondiera "el club ha hecho sus evaluaciones y ha elegido este uniforme; le guste o no, para la temporada en curso el uniforme no cambiará". En el primer caso - en el que A1 afirma que el uniforme es horrible y A2 afirma, en cambio, que el uniforme es hermoso - estamos en medio de un desacuerdo meramente estético sobre el juego de fútbol. En este caso, la práctica objeto (el juego de fútbol) permanece completamente inerte. Desde un punto de vista estético, este es un desacuerdo intradisciplinario, mientras que desde el punto de vista de la práctica objeto el desacuerdo es metadisciplinario. En cambio, cuando el técnico de la Juventus (A3) nota que "el club ha hecho sus evaluaciones y ha elegido este uniforme; le guste o no, para la temporada en curso el uniforme no cambiará", ocurre algo más complejo. A1 sostiene que el argumento estético es una razón para cambiar el uniforme, mientras que A3 sostiene que el argumento estético es irrelevante (al menos para la temporada actual) desde la perspectiva del juego de fútbol. En este caso, A1 afirma la relevancia de la proposición estética para el juego de fútbol, mientras que A3 observa que, desde el punto de vista del juego de fútbol, la proposición estética de A1 no es

6 Para una definición más elaborada, véase Koczogh (2013), pp. 211-222. Koczogh parte de la distinción, utilizada por SEARLe, entre cuatro condiciones de los actos de habla (preparatorias, proposicionales, de sinceridad y esenciales). Me limito a una definición basada en una versión ligeramente modificada de la primera condición preparatoria y esa condición. La razón es que dos hablantes pueden estar en desacuerdo sin saberlo y además el desacuerdo puede ser ficticio, porque las partes creen que están en desacuerdo, pero en realidad no lo están. 
relevante. Llamaré a este desacuerdo "desacuerdo interdisciplinario en sentido estricto". De manera general:

Desacuerdo metadisciplinario: A1 afirma (o implica) una proposición $P 1(P x)$ mientras que A2 afirma (o implica) una proposición diferente de $P 1(P x)$;

Desacuerdo interdisciplinario en sentido estricto: A1 afirma (o implica) una proposición $P 1(P x)$ como una razón para avalar $P x$ en la práctica objeto mientras que A2 afirma (o implica) que $P 1(P x)$ no cuenta como una razón para avalar a $P x$ en la práctica objeto.

Nótese que un desacuerdo metadisciplinario podría combinarse con un desacuerdo interdisciplinario en sentido estricto, generando así un desacuerdo interdisciplinario complejo. Volviendo a la relación entre la estética y los uniformes de fútbol, puede estarse en desacuerdo respecto de la belleza del uniforme de la Juventus y también respecto de la relevancia de este desacuerdo desde el punto de vista del juego del fútbol. Piénsese, por ejemplo, en el caso de alguien que afirma que el equipo con el uniforme más hermoso debería comenzar con una ventaja de goles.

En este ensayo, enfoco mi atención en los desacuerdos interdisciplinarios en sentido estricto. La razón es que, si no aclaramos cómo resolver estos desacuerdos de manera tal que una metaproposición resulte relevante para la práctica objeto, un posible desacuerdo metadisciplinario se mantiene irrelevante para la práctica objeto. Una vez aclarado lo que es un desacuerdo interdisciplinario en sentido estricto, consideremos algunos de ellos en los que las proposiciones económicas ${ }^{7}$ tienen como objeto el derecho:

1. "La investigación conductual muestra que [...] contrariamente a la suposición [del Tribunal de Justicia de la Unión Europea] de que el consumidor promedio está 'razonablemente bien informado y [es] razonablemente atento y cauteloso', los consumidores a menudo están mal informados y tienen dificultad para aprovechar plenamente las oportunidades del mercado": 8

2. "Desde un punto de vista económico, los remedios contractuales son deseables si constituyen un conjunto de reglas que generen in-

7 Cabe señalar que las siguientes son, en rigor, enunciados. Sin embargo, estos enunciados son lo suficientemente claros como para afirmar que expresan proposiciones que son prima facie coincidentes con los enunciados de los que son el contenido proposicional.

8 Almeida et al. (2016), p. 9. 
centivos eficientes ${ }^{9}[\ldots]$. Tradicionalmente, la teoría jurídica no da mucho peso a los incentivos eficientes. Los remedios contractuales, más bien, apuntan a ofrecer protección a la parte que es perjudicada como consecuencia del incumplimiento de su obligación por parte del otro contratante. Sin embargo, la perspectiva jurídica no entra en conflicto con la perspectiva económica"; ${ }^{10}$

3. "[El derecho de] la competencia se preocupa principalmente por la eficiencia económica y apunta a aumentar el bienestar general de la sociedad por medio de mantener la rivalidad entre las empresas". ${ }^{11}$

La Proposición 1 es una afirmación de un informe publicado por el Joint Research Centre, "el servicio científico interno de la Comisión Europea". ${ }^{12} \mathrm{La}$ Proposición 1 sostiene que el paradigma del consumidor razonable introducido por el Tribunal de Justicia Europeo en su jurisprudencia es contrario a los resultados de la investigación conductual. Este es un tipo de desacuerdo descriptivo: se refiere a cómo son realmente los consumidores y, por lo tanto, cómo es realmente el mundo. La Proposición 2, en cambio, contrasta las opiniones económica y jurídica respecto de la finalidad de los remedios contractuales. Desde el punto de vista económico, este objetivo es proveer incentivos eficientes. Desde la perspectiva jurídica, sin embargo, el objetivo es proteger frente el incumplimiento. Al mismo tiempo, sin embargo, no existe un conflicto práctico entre las dos perspectivas. En este caso existe un desacuerdo sobre el propósito de los remedios contractuales: incentivos eficientes para la economía y protección frente a la infracción jurídica. Incidentalmente, sin embargo, las dos posturas convergerían en torno a cuáles sean las reglas deseables. Por último, la Proposición 3 se refiere a una práctica jurídica, el derecho de la competencia. El lenguaje usado sugiere claramente que se trata de una proposición económica, pero, a diferencia de las Proposiciones 1 y 2 , no existe desacuerdo. Sin embargo, considérese también la siguiente proposición:

9 Schweizer define una asignación eficiente como "si no existe otra asignación posible que sea preferida por todas las partes involucradas".

10 SCHWEIZER (2009), pp. 1-18; p. 2.

11 CSERES (2005), p. 405. En la cita original, el tema es la competencia. Sin embargo, del contexto se desprende claramente que el autor tiene la intención de referirse al derecho de la competencia.

12 Almeida et al. (2016), p. 1. La del Joint Research Centre no es la posición oficial de la Comisión Europea. Sobre el impacto de la economía conductual en la teoría económica de la protección del consumidor, ver EsPosito (2017), pp. 193-216. 
3' "El objetivo del derecho de la competencia es proteger el mercado como medio de mejorar el bienestar de los consumidores". ${ }^{13}$

Es claro que existe un desacuerdo entre quienes, como Cseres (y muchos economistas), apoyan la Proposición 3 y quienes, como el Director General de la Comisión Europea Lowe, apoyan la Proposición 3’. Nuevamente, el desacuerdo se refiere al propósito del derecho.

Ahora, imagínese que usted es un juez. ¿Qué ocurre si alguien afirma que la Proposición 1 es una razón para decidir un caso de manera distinta del precedente relevante? ¿O si se trata de una compleja discusión en la que, por ejemplo, alguien invoca la aplicación de una indemnización punitiva para proveer incentivos eficientes, a pesar de que esto vaya más allá de la protección del demandante frente al incumplimiento contractual del demandado? ¿O, más aun, si se critica una aplicación del derecho de la competencia porque reduce el bienestar general incluso si aumenta el bienestar de los consumidores? En todos estos casos, y otros similares, el problema a abordar es el siguiente: ¿Existe alguna razón para tener en cuenta la proposición económica en la discusión jurídica o puede considerarse irrelevante?

Intuitivamente, el tercer caso parece el más sencillo. El derecho de la Unión Europea ha adoptado una postura distinta de la Proposición 3: La Proposición 3, por tanto, es jurídicamente irrelevante. Sin embargo, surge una duda: si es verdad $^{14}$ que para los economistas la competencia debe maximizar el bienestar general, ¿por qué el derecho de la UE ha adoptado una postura diferente?

Quizás los juristas no han comprendido plenamente el objetivo económico de la competencia en el mercado. O podría ser que, visto bajo su mejor luz como recomienda Dworkin - el derecho de la competencia apunta a maximizar el bienestar general. Incluso con la Proposición 2, las cosas no son mucho mejores. Si existe una convergencia entre la función de proteger contra el incumplimiento y proveer incentivos efectivos, en general, ${ }^{15}$ ¿no podría ser que los miembros de jurados estén confundidos y que realmente estén actuando para formular in-

13 Lowe (2007). Para una investigación mucho más profunda del propósito del derecho de la libre competencia de la UE, ver Hildebrand, (1998).

14 Aunque esta posición es claramente mayoritaria en la teoría económica, existen posiciones alternativas que tienden a mover el enfoque desde el bienestar general al bienestar del consumidor. Discuto esto en Esposito y Grundmann (2017) y en EsPosito y DE Almeida (2018).

15 Por "convergencia" quiero decir que se llega a la misma conclusión, pero sobre la base de razones diferentes. 
centivos eficientes? ¿Qué saben los jueces del Tribunal de Justicia de la Unión Europea sobre el comportamiento real de los consumidores? ¿Por qué deberíamos confiar en su opinión en lugar de la de los científicos del comportamiento que han estudiado científicamente el comportamiento del consumidor por años?

Cuando se tienen dudas como estas, se duda de que las proposiciones económicas relativas al derecho puedan utilizarse para avalar proposiciones jurídicas. Como veremos mejor en la siguiente sección, este es un tipo particular de desacuerdo: un desacuerdo que es tanto interdisciplinario en sentido estricto como teórico en el sentido hecho famoso por Dworkin - es decir, un desacuerdo teórico interdisciplinario.

\section{EL PUNTO DELICADO DE LOS DESACUERDOS TEÓRICOS Y SU RELACIÓN CON LOS DESACUERDOS INTERDISCIPLINARIOS}

En Law's Empire, Dworkin sostuvo que el positivismo jurídico no puede dar cuenta adecuadamente de lo que, según él, es un aspecto central de la práctica jurídica, a saber, los desacuerdos teóricos. Los desacuerdos teóricos son desacuerdos sobre las condiciones que deben cumplirse para que una proposición de derecho sea verdadera. ${ }^{16}$ Deben distinguirse de los desacuerdos empíricos, que ocurren cuando hay acuerdo sobre qué condiciones deben cumplirse, pero no está claro si estas han ocurrido. Más que el concepto de verdad, prefiero utilizar el concepto epistémico de aval [warrant], de acuerdo con la estipulación propuesta por Susan Haack. La autora identifica tres componentes del aval ofrecido por la evidencia en favor de una afirmación: grado de apoyo [supportiveness], seguridad independiente [independent security] y comprehensión [comprehensiveness]. ${ }^{17}$ El concepto de aval de Haack me parece interesante por tres razones. En primer lugar, ella presenta una teoría general, aplicable a cualquier tipo de evidencia y afirmaciones. Esto es particularmente útil aquí, donde el interés radica en la relación entre distintas disciplinas. En segundo lugar, el concepto de aval es más práctico que el de la verdad. Por ejemplo, incluso si se considerara que es incierto que la Tierra gire alrededor del Sol, aun así podría suponerse que la afirmación de que la Tierra gira alrededor del Sol está mejor avalada por la evidencia disponible que la afirmación de que es el Sol el que gira alrededor de la Tierra. Finalmente, el concepto de aval desplaza la atención desde la afirmación o proposición avalada al argumento que la avala. Esto, como veremos mejor a

16 DWORKIN (1986), pp. 4-5.

17 HAACK (2015), pp. 19-21. 
continuación, es exactamente el tipo de perspectiva que permite - o al menos esta es la posición defendida en este ensayo - dar cuenta del problema de la traducción interdisciplinaria:

desacuerdo teórico: A1 afirma (o implica) que una proposición de derecho está avalada si ocurre la condición $C$ y A2 afirma (o implica) que una proposición de derecho está justificada si ocurre una condición diferente de $C$.

Según Dworkin, el problema con el positivismo jurídico es que considerar el derecho como una práctica convencional distorsiona lo que sucede cuando los abogados están en desacuerdo respecto lo que la ley establece. Si el derecho es un conjunto de hechos sociales convencionales, cuando hay desacuerdo acerca de cuáles son esos hechos convencionales, entonces no hay derecho. Así, los juristas estarían equivocados o serían intelectualmente deshonestos al afirmar que existe un hecho social convencional en apoyo de sus afirmaciones sobre lo que el derecho establece. Por lo tanto, el positivismo jurídico no logra dar cuenta del aspecto [face value] de la práctica jurídica. Como es bien sabido, en 2007 Shapiro sostuvo que los positivistas no habían ofrecido una respuesta adecuada a esta crítica. ${ }^{18}$

En el campo positivista, se pueden distinguir dos estrategias principales para replicar a Dworkin. ${ }^{19}$ Una la debemos a Leiter. El autor, en pocas palabras, formula dos réplicas. En primer lugar, el fenómeno de los desacuerdos teóricos no es en absoluto ubicuo, sino que concierne solo a la parte superior de la pirámide jurídica. Por lo tanto, no es un aspecto central de la práctica jurídica. Por consiguiente, considerando que el enfoque positivista general logra dar cuenta de la práctica jurídica mejor que la teoría de Dworkin, aquél sería preferible incluso si fuera incapaz de ofrecer una explicación convincente de los desacuerdos teóricos. En segundo lugar, los desacuerdos teóricos se explican bastante bien en términos de error intelectual y deshonestidad. ${ }^{20}$ La segunda estrategia se debe en particular a Shapiro y Ratti (y a sus coautores Dolcetti y Ferrer). Estos positivistas enfocaron su atención en la centralidad de la interpretación jurídica. ${ }^{21}$ Para subrayar la centralidad de la interpretación, Shapiro incluso ha abandonado

18 Shapiro (2007), pp. 22-55.

19 Para una descripción más precisa de las réplicas positivistas, así como la formulación de una respuesta pluralista adicional, ver RAMíREz (2016), pp. 11-32.

20 LeITER (2009), pp. 1215-1250.

21 Un aspecto digno de mención de esta respuesta - aunque es irrelevante para los propósitos presentes - es que esta segunda línea de argumentación está dispuesta a aceptar la observación empírica de que los desacuerdos teóricos son un fenómeno central. 
la expresión "desacuerdos teóricos" en favor de las expresiones "desacuerdos interpretativos" y, luego, "desacuerdos (meta-)interpretativos" en Legality. ${ }^{22}$ Ciertamente no hay grandes diferencias entre las posiciones de estos autores. Ratti y Ferrer sostienen incluso que Shapiro ofrece una teoría normativa en lugar de una descriptiva - lo que sería, además, incompatible con el positivismo excluyente, en la versión previamente defendida por Shapiro. ${ }^{23}$ Sea como fuere, estos autores están de acuerdo en que el uso de argumentos o cánones interpretativos juega un papel central en la explicación de los desacuerdos teóricos. De hecho, la explicación de Dworkin - basada en la interpretación como una actividad orientada a mostrar al derecho bajo su mejor luz - también se enfoca en el tema de la interpretación. Por lo tanto, existe un amplio acuerdo teórico de que los argumentos interpretativos juegan un papel importante en la identificación de estándares jurídicos. En otras palabras, los argumentos interpretativos son importantes para avalar una proposición jurídica.

Volvamos ahora a la cuestión de la relevancia para el derecho de los acuerdos interdisciplinarios; es decir, a la pregunta “¿bajo qué condiciones está justificado un abogado en ignorar la perspectiva económica?". Sobre la base de las consideraciones desarrolladas más arriba sobre la relación entre los argumentos interpretativos y el aval de las proposiciones jurídicas los abogados están interesados en formular argumentos interpretativos capaces de avalar proposiciones jurídicas. Por lo tanto, la respuesta obvia parece ser que un desacuerdo interdisciplinario que no es también un desacuerdo teórico - por lo tanto: un desacuerdo puramente interdisciplinario - es un desacuerdo irrelevante para el jurista que quiere avalar proposiciones jurídicas. Este jurista, de hecho, está interesado en desacuerdos interdisciplinarios que son tanto interdisciplinarios como teóricos, es decir, desacuerdos teóricos interdisciplinarios. Lo que le interesa es si acaso la proposición económica es relevante o no para avalar una proposición jurídica: ${ }^{24}$

desacuerdo teórico interdisciplinario: A1 afirma (o implica) que la proposición $P 1(P x)$ es parte de la condición $C$ para avalar $P x$ en la práctica objeto mientras que $\mathrm{A} 2$ afirma (o implica) que la proposición $P 1(P x)$ no es parte de la condición $C$.

22 SHAPIRO (2011).

23 Véanse en particular las críticas de Ratti y Ferrer a Shapiro en RatTi y FERRER (2013), pp. 169-186.

24 Por otro lado, habría un desacuerdo empírico-interdisciplinario si no se tuviera la certeza de que la proposición interdisciplinaria, de estar garantizada, verificaría la condición $C$. En el desacuerdo teórico interdisciplinario, en cambio, el problema se refiere a la relevancia de la proposición interdisciplinaria para la ocurrencia de la condición $C$. 
El problema ahora pasa a ser comprender cuándo un desacuerdo es puramente interdisciplinario y cuándo, en cambio, da lugar a un desacuerdo teórico interdisciplinario. Para aclarar esto, debemos enfocarnos en el problema de traducir las proposiciones de la economía al derecho.

\section{EL PROBLEMA DE LA TRADUCCIÓN, ESTO ES, CUANDO LO QUE LOS ECONOMISTAS PIENSAN IMPORTA}

En esta sección, busco responder dos preguntas relacionadas con la distinción entre desacuerdos puramente interdisciplinarios y teóricos interdisciplinarios, es decir, el problema de traducir proposiciones interdisciplinarias desde la economía al derecho: $a$ ) “¿cuál es el problema?”; $b$ ) “cómo se le enfrenta?”.

Empiezo comentando algunas de las tesis presentadas por Papayannis que son relevantes para el tema. Él cree que cuando el derecho usa términos económicos, por ejemplo, precio predatorio, "el significado de 'precio predatorio' parece depender indudablemente de consideraciones propias de la teoría económica". ${ }^{25}$ Papayannis parece estar a favor del uso de un argumento literal basado en el conocimiento experto, según el cual el significado de un término es el significado de ese término de acuerdo con los expertos (en este caso, los economistas). No olvidemos el problema - bien ejemplificado por la turbulenta historia de los testimonios expertos en los Estados Unidos ${ }^{26}$ - de los desacuerdos entre expertos. ${ }^{27}$ Considero esta observación sobre términos técnicos infundada. El autor parece establecer una jerarquía interpretativa arbitraria en la que prevalece el argumento literal basado en el conocimiento experto. Esta jerarquía arbitraria termina negando la distinción entre explicaciones conceptuales internas y explicaciones funcionales que el propio Papayannis discute latamente.

Me parece que la posición de Papayannis se explica por el hecho de que acepta la Proposición 3, o una muy similar, como ejemplo de una explicación conceptual interna. Papayannis llama explicaciones conceptuales internas a aquellos análisis que despliegan conceptos pertenecientes a una práctica; y llama funcionales a aquellas externas a la práctica porque son realizadas utilizando conceptos típicos de otra práctica. El punto nodal es que el significado económico de un término se establece típicamente dentro de un análisis funcional. Más precisamente, los economistas suelen definir el significado de términos como

25 PAPAYANnis (2013), pp. 69-110; p. 73.

26 Sobre este punto, véase HaAcK (2015), pp. 157-205.

27 PAPAYANNIS (2013), pp. 73-74. 
"precios predatorios" o "fallas de mercado" en un análisis en el que asignan una función ${ }^{28}$ a la práctica modelada. Por lo tanto, cuando el análisis funcional no llega a ser un análisis conceptual interno - en los términos de este ensayo: cuando produce proposiciones metadisciplinarias puras - aceptar el significado económico de un término exige aceptar la función asignada al objeto por los economistas. Esto significa traducir implícitamente la función económica a un concepto jurídico. Por ejemplo, imagínese que un juez rechaza la Proposición 3 debido a la Proposición 3'. Sin embargo, el mismo juez acepta un significado económico de "precio predatorio" que está justificado por la misma Proposición 3. La norma resultante sería funcional a la maximización del bienestar general. Esto significa que el propósito de la disposición, tal como se interpreta, no sería maximizar el bienestar de los consumidores, sino maximizar el bienestar general.

Para explicar el error que (supuestamente) Papayannis comete y para avanzar en la investigación, se puede partir de la conclusión que él extrae del ejemplo sobre el significado del "precio predatorio": "los modelos económicos tienen incidencia en la interpretación de términos con contenido económico. Y que un término tenga un contenido económico depende de cómo se haya desarrollado la práctica a su respecto". ${ }^{29}$ Me parece que el problema central es que la práctica que determina si un término jurídico tiene contenido económico no puede ser la práctica económica - para ella todos los términos tienen contenido económico -, sino que debe ser la práctica jurídica. En el lenguaje presentado en la sección $\S$ 2 , al construir su argumento en favor del uso jurídico del significado económico del "precio predatorio", Papayannis acepta apresuradamente que una proposición metadisciplinaria es una razón para avalar una proposición jurídica. ¿Cómo comete este agudo erudito ese error? Creo que la razón es que, para Papayannis, contingentemente no hay ningún desacuerdo interdisciplinario sobre el hecho de que la competencia está dirigida a lograr la "maximización de la riqueza", ${ }^{30}$ lo que - en la terminología de Richard Posner - coincide con la Proposición 3. Sin embargo, como se ha señalado, existe un desacuerdo interdisciplinario. Sobre esta base, la tesis de Papayannis sobre el significado del "precio predatorio" se explica como la consecuencia de aceptar la traducción de la Proposición 3 desde la economía al derecho. Por lo tanto, el argumento literal basado en el conocimiento experto se justifica por un argumento teleológico interdisciplinario implícito, consistente en el hecho de que la función económica de la competencia y su propósito jurídico coinciden. Esto es también indicativo de una limitación adicional del análisis de

28 La función de una práctica es típicamente representada por la función (matemática)-objetiva que busca maximizar su valor.

29 PAPAYANNis (2013), p. 74.

30 PaPAYANnis (2013b), pp. 89-128; p. 109. 
Papayannis, que nos permite identificar un aspecto importante del problema de la traducción, a saber, la posibilidad de traducciones implícitas.

Papayannis cree que la clasificación de una explicación como funcional implica que esta función es "opaca a los participantes". ${ }^{31}$ No considera la posibilidad de que una proposición interdisciplinaria sea tan obvia que sea percibida por los participantes en una práctica como interna a su propia práctica, incluso si no se presenta como tal. Llamaré al fenómeno en cuestión "traducción implícita". Como acabamos de ver, creo que el argumento de Papayannis sobre "precio predatorio" es el resultado de una traducción implícita (errónea). El error adicional del autor es entonces haber generalizado esta conclusión a todos los términos económicos.

Consideremos una tesis positivista jurídica clásica como la del derecho (moderno) como la unión de reglas primarias y secundarias. ${ }^{32}$ Papayannis considera el análisis de Hart de las reglas secundarias como un ejemplo de análisis conceptual funcional pero no interno. ${ }^{33}$ Sobre esta base, concluye que es erróneo considerar el análisis de la función de las reglas secundarias como interno. Como es bien sabido, para Hart, las reglas primarias son aquellas que establecen los criterios de conducta de los sujetos. Las reglas secundarias, por otra parte, corrigen los defectos en un sistema jurídico que consista únicamente en reglas primarias. Estos defectos son falta de certeza, la naturaleza estática y la ineficiencia. Un sistema de solo reglas primarias es incierto porque carece de reglas para reconocer las reglas primarias; es estático porque carece de reglas para modificar las reglas primarias; y, por último, es ineficiente porque carece de reglas para resolver las controversias relativas a las infracciones de las reglas primarias. El tipo de regla secundaria que aborda la incertidumbre es la regla de reconocimiento; la que aborda la naturaleza estática es la regla del cambio; finalmente, la ineficiencia es abordada por un tipo de regla secundaria que Hart llama regla de adjudicación. Este no es el lugar para estudiar esta clasificación en profundidad. ${ }^{34}$

Lo que nos interesa aquí es sugerir que los defectos identificados por Hart son problemas tan típicos para los participantes de la práctica jurídica que parece artificial que un jurista los considere como intereses o valores externos a la práctica

31 Papayannis (2013b), p. 126.

32 HART (1994), pp. 91-97.

33 PAPAYANNIS (2013b), pp. 125-127.

34 Ver, por ejemplo, Shapiro (2009), pp. 235-268 y Pino (2015), pp. 183-201. Creo que la propuesta de Pino de considerar el estándar de reconocimiento como un criterio tanto de aplicabilidad como de validez, y de vincular su estudio a la teoría del razonamiento jurídico está muy en línea con la perspectiva adoptada en este ensayo. 
legal. De hecho, los abogados están claramente comprometidos con la certeza jurídica, pero también con la adaptabilidad del derecho, así como con evitar el desperdicio de recursos asignados a la resolución de disputas. En relación con el sistema jurídico italiano, estos intereses o valores son ejemplificados por la reserva de ley y la prohibición de la analogía en el derecho penal (certeza), por las reglas que rigen el proceso legislativo y la autonomía privada (adaptabilidad), y por el principio de buen desempeño de la administración pública (eficiencia). Me parece que estos intereses y valores son tan obvios para el jurista que él o ella claramente los considera relevantes.

Nuevamente en referencia a la teoría de Hart, valen consideraciones similares respecto de los cinco truismos en los que se basa el "contenido mínimo del derecho natural". Los truismos son "verdades elementales referentes a los seres humanos, a su circunstancia natural, y a sus propósitos", que "suministran una razón para que, dada la supervivencia como objetivo, el derecho y la moral deban incluir un contenido específico". ${ }^{35} \mathrm{El}$ hecho de que Hart identifique estas tesis como truismos indica claramente que son tan obvios que parece difícil negarlas.

Lejos de la tradición positivista, un fenómeno similar se encuentra en la tesis de Alexy de que el valor de los principios varía de acuerdo con el supuesto económico de la utilidad marginal decreciente. ${ }^{36}$ Una vez más, parece totalmente razonable pensar que pequeños aumentos en el grado de satisfacción de un principio que actualmente está poco satisfecho por parte del sistema jurídico son, ceteris paribus, más importantes que aumentos menores en el grado de satisfacción de un principio que actualmente está satisfecho en gran medida.

En un nivel más profundo, aunque Papayannis admite la posibilidad de la traducción, no profundiza en el mecanismo que lo hace posible. ${ }^{37}$ Por el contrario, parece creer que el problema de la traducción es de poco interés para el jurista porque no le permite obtener "ningún conocimiento adicional respecto del provisto por una explicación conceptual interna". ${ }^{38}$ Evidentemente, esta no es la perspectiva adoptada en este ensayo: la traducción de las proposiciones interdisciplinarias desde la economía al derecho es un aspecto importante de la

35 HART (1994), p. 193.

36 Alexy (2002), pp. 102-109. Para un análisis exhaustivo de las conexiones entre la teoría de Alexy y los conceptos económicos, ver Esposito (2018), pp. 185-210.

37 Papayannis (2013), pp. 78-79. Sin embargo, en Papayannis (2013b), afirma que las "explicaciones externas [...] se basan en una metodología que imposibilita determinar el contenido del derecho", p. 121.

38 PAPAYANNis (2013), p. 83. 
relación entre el derecho y la economía. Por lo tanto, es necesario especificar con mayor claridad cómo es posible esta actividad.

Ya nos hemos encontrado con la tesis de que algunas proposiciones interdisciplinarias son tan obvias que pueden ser traducidas implícitamente desde la práctica económica a la práctica jurídica. Sin embargo, no es claro aún qué se hace exactamente con la "traducción" o cómo se hace.

Un punto de partida útil para reflexionar sobre esta cuestión la ofrece un debate entre Patterson y Craswell sobre el uso de argumentos económicos para la resolución de disputas relativas a contratos incompletos. Patterson comienza presentando la distinción entre "proposiciones de" y "proposiciones sobre" una práctica. La distinción es importante, observa, porque las proposiciones de una práctica son avaladas de acuerdo con las reglas de aval de esa práctica. Por lo tanto, una proposición económica sobre el derecho - como la Proposición 1 - debe ser avalada de acuerdo con las reglas de aval de la práctica económica. Cuando esta proposición se utiliza en la práctica jurídica, esto no es suficiente: la proposición debe estar avalada de acuerdo con las reglas de aval de la práctica jurídica. Si esto no es el caso, la proposición no es jurídicamente relevante.

Patterson también sostiene que la principal forma de formular una proposición económica relativa al derecho jurídicamente relevante es considerarla como un argumento prudencial, ${ }^{39}$ es decir, un argumento consecuencialista. ${ }^{40}$ En respuesta a Patterson, Craswell concede que los economistas deberían involucrarse más en tratar de traducir proposiciones económicas, pero también añade dos aclaraciones importantes. En primer lugar, la conexión entre la proposición económica y el argumento prudencial sugiere que cualquier proposición económica relativa al derecho debe concebirse como precedida de la "premisa implícita" de que tal tesis debe entenderse como un argumento prudencial. En segundo lugar, Craswell cree que Patterson es demasiado pesimista sobre la posibilidad de traducir proposiciones económicas. ${ }^{41}$ De hecho, la tesis doctoral de Habib - de la que Patterson fue el supervisor - ofrece un claro ejemplo de esta segunda aclaración.

39 PATterson (1993), pp. 235-288; p. 278. Para una reconstrucción del debate y su desarrollo con referencia a la distinción entre los argumentos deónticos y consecuencialistas, ver CSERne (2011), pp. 31-54.

40 Patterson (1993), p. 276. Más recientemente, Patterson definió el "argumento prudencial" como un argumento destinado a pesar o evaluar las consecuencias (en términos de “costos") de una determinada regla, Patterson (2005), p. 694.

41 Craswell (1993), pp. 289-302. 
Habib quiere llamar nuestra atención sobre el hecho de que hay una forma de pensar característica del derecho privado que está siendo progresivamente marginada. Entre otras tesis, observa que el derecho privado permite la traducción de proposiciones económicas a proposiciones de derecho privado. Como ejemplo, Habib analiza la proposición económica presentada por Eisenberg de que existe un tipo de "estructura promisora", que Habib llama promesa interesada, caracterizada por el hecho de que una parte hace una promesa que aumenta la probabilidad del intercambio, pero tal promesa no requiere a cambio ni una promesa ni un acto. ${ }^{42}$

Habib muestra que, si bien Eisenberg no defiende con argumentos jurídicos la obligatoriedad (jurídica) de una promesa interesada, es posible construir esos argumentos - $\mathrm{o}$ al menos este es el intento de Habib. Más precisamente, Habib argumenta en gran detalle que existe un intercambio de valores entre los promitentes y, como resultado - este es el punto nodal - la supuesta obligación hacia el otro promitente de quien hace la promesa tiene una causa conmutativa. Por lo tanto, la proposición "una promesa interesada es fuente de obligaciones" debe considerarse como una proposición jurídica avalada. En otras palabras, Habib sostiene que, en el derecho privado, cualquier interacción con una causa sinalagmática es fuente de obligaciones y que, como resultado, una promesa interesada es fuente de obligaciones bajo el derecho privado. En este punto parece útil aclarar el fundamento que justifica la conclusión de que la promesa interesada es una fuente jurídica de obligaciones:

L1 la causa conmutativa es fuente de obligaciones;

L2 un intercambio sinalagmático tiene una causa conmutativa;

E1 en una promesa interesada hay un intercambio sinalagmático;

C la promesa interesada es fuente de obligaciones.

En el razonamiento de Habib, las dos primeras premisas (L1) y (L2) y la conclusión (C) son jurídicas, mientras que solo la tercera premisa (E1) es económica. El razonamiento muestra tres aspectos importantes del problema de la traducción. En primer lugar, el hecho de que se traduzca una proposición no significa que la conclusión del razonamiento sea jurídicamente correcta porque siempre se puede dudar de la validez jurídica de las premisas. Por ejemplo, se podría rechazar (L1) o (L2) o sostener que 'intercambio sinalagmático' tiene significados diferentes en (L2) y (E1). Pero si se aceptan las premisas, la lógica exige que se acepte la conclusión. En segundo lugar, una traducción también

42 Навів (2016), еsp. pp. 35-51. 
puede tener lugar a través de un argumento - como el de Habib, que es conceptual - muy distinto de un argumento prudencial. En tercer lugar, y en general, la traducción se produce cuando una proposición económica se incorpora en el aval de una proposición jurídica.

Antes de terminar, parece útil recordar el modelo de razonamiento judicial introducido por Wróbleski. ${ }^{43}$ Wróbleski comienza del conocido modelo de silogismo judicial introducido por Beccaria, en el que la decisión del caso concreto se deriva deductivamente de una premisa mayor jurídica y de una premisa menor fáctica:

1. premisa mayor jurídica;

2. premisa menor fáctica;

3. decisión.

Wróbleski observa que el razonamiento jurídico no puede reducirse completamente a esta inferencia, ya que excluye una serie de importantes operaciones responsables de la justificación de las premisas que son realizadas por los juristas. Por lo tanto, el modelo debe ser enriquecido con la etapa de justificación de las premisas:

1. premisa mayor jurídica $\leftarrow$ justificación externa jurídica;

2. premisa menor fáctica $\leftarrow$ justificación externa fáctica;

3. decisión.

No nos interesa aquí investigar si existe una relación y, en caso afirmativo, cuál, entre la justificación de la premisa fáctica y la jurídica. Lo que hay que notar es que la traducción desde la práctica económica a la práctica jurídica se produce solo cuando la proposición económica pasa a formar parte de la justificación externa jurídica ${ }^{44}$ En efecto, solo los elementos de la justificación jurídica externa pueden ser pertinentes para avalar la premisa mayor jurídica. Si la traducción no tiene lugar, la proposición económica se permanece completamente irrelevante para propósitos de identificar el derecho aplicable en el caso concreto.

43 WróbleSKi (1992). Para una revisión de la evolución del debate, ver CANALE (2013), pp. 316-351.

44 Uno también debería preguntarse si las proposiciones económicas pueden usarse tanto para identificar fuentes como para interpretarlas, pero un análisis profundo no es posible en el espacio de este ensayo. 
A la luz de las consideraciones anteriores, las preguntas que abren esta sección admiten las siguientes respuestas:

1. ¿Cuál es el problema de la traducción desde la economía al derecho? El problema es determinar si una proposición económica es relevante para avalar proposiciones jurídicas;

2. ¿Cómo enfrentamos el problema de la traducción desde la economía al derecho? El problema se enfrenta integrando la proposición económica en la justificación externa jurídica de una proposición jurídica, es decir, sosteniendo que la práctica jurídica permite incorporar la proposición económica en el razonamiento de identificación de una proposición jurídica.

Es importante señalar que la solución del problema de la traducción interdisciplinaria no significa que la proposición jurídica respaldada por la proposición económica esté suficientemente avalada como para ser aplicada, sino solo que la proposición económica es relevante para la justificación de la regla que ha de aplicarse. De hecho, incluso después de la traducción es posible estar en desacuerdo respecto de que la proposición jurídica respaldada por la proposición económica es la que ha de aplicarse. En particular, en caso de desacuerdo respecto de las condiciones que deben cumplirse para que la proposición jurídica esté avalada, existirá un desacuerdo teórico. Sin embargo, dado que se ha superado el desacuerdo sobre la relevancia jurídica de la proposición económica, el desacuerdo ya no es uno teórico interdisciplinario.

Sobre la base de estas reflexiones, creo que la primera tesis de este ensayo está debidamente avalada: los desacuerdos entre la economía y el derecho pueden dar lugar a desacuerdos teóricos interdisciplinarios si y solo si las proposiciones económicas se traducen en la justificación externa de las proposiciones jurídicas.

\section{DESACUERDOS SOBRE LA RELACIÓN ENTRE EL DERECHO Y LA MORAL COMO PROBLEMA DE TRADUCCIÓN}

En la sección anterior se vio que el problema de la traducción desde la economía al derecho es un problema sobre la relevancia de una proposición económica para la justificación externa de una norma jurídica y que la solución a este problema depende de la forma en que se desarrolle la práctica jurídica en un caso dado. Una objeción - podría decirse - "identitaria" consiste en afirmar que este tipo de información no cae en el ámbito de los temas que la teoría y la filosofía del derecho están llamadas a tratar. Personalmente, creo que esta ob- 
servación es conceptualmente poco caritativa e inexacta desde un punto de vista historiográfico, pero no quiero rechazarla en ese nivel. Prefiero mostrar cómo algunas proposiciones teóricas bien conocidas sobre la relación entre el derecho y la moral pueden describirse efectivamente como un problema de taducir proposiciones interdisciplinarias desde la moral al derecho. Me refiero en particular a algunas versiones de las tesis de la conexión (C) y de la separabilidad (S) entre el derecho y la moral: ${ }^{45}$

C1. tesis iusnaturalista fuerte: el derecho inmoral no derecho;

C2. tesis iusnaturalista débil: el derecho intolerablemente inmoral no es derecho;

C3. tesis neoconstitucionalista: en los estados constitucionales, el derecho inmoral no es derecho;

S1. tesis del positivismo jurídico incluyente: el derecho inmoral puede puede ser derecho;

S2. tesis positivista excluyente débil: el derecho puede permitir la aplicación de estándares morales;

S3. tesis positivista excluyente fuerte: el derecho inmoral es derecho.

Se puede apreciar inmediatamente que las dos tesis extremas, es decir, la tesis iusnaturalista fuerte y la tesis positivista excluyente fuerte, son dos opiniones categóricas pero opuestas sobre la relación entre el derecho y la moralidad. La primera tesis siempre admite esta relación como relevante para el derecho y, de hecho, también se le denomina tesis de la conexión necesaria entre el derecho y la moral. La segunda tesis siempre niega que esta relación sea relevante para el derecho y, de hecho, también se le denomina tesis de la separación necesaria entre el derecho y la moral. Al mismo tiempo, las cuatro tesis intermedias admiten formas limitadas de conexión entre el derecho y la moral: para la tesis iusnaturalista débil, el límite consiste en el umbral de la tolerabilidad; para la tesis neoconstitucionalista, se encuentra en el carácter constitucional del sistema jurídico; para el positivismo incluyente, la pregunta admite una respuesta fáctica. Finalmente, incluso para la tesis positivista excluyente débil, la pregunta admite una respuesta fáctica: debe observarse si acaso en un sistema jurídico dado el derecho permite la aplicación de la moral.

45 Para una discusión de estas y otras tesis sobre el tema de la conexión y la separabilidad, ver, por ejemplo, BARBERIS (2011), pp. 55-93. 
Hasta este punto, he resumido una serie de posiciones típicamente discutidas en la teoría jurídica en los términos en que se suelen discutir. Ahora quiero mostrar que estas consideraciones pueden reformularse en términos de la relevancia de la moral para la justificación externa de las reglas jurídicas. Si este es el caso, las tesis mencionadas anteriormente son respuestas distintas a la pregunta "¿bajo qué condiciones se justifica que el jurista ignore la perspectiva moral?" y por tanto están relacionadas con el problema de la traducción, esta vez desde la moral al derecho.

Para comprobarlo, considérese el caso en que un abogado sostiene que "la norma N, invocada por la otra parte como la norma jurídica a aplicar en el presente caso, es contraria a la norma moral M". La reacción de una participante de la práctica jurídica a esta afirmación nos permite hacer inferencias respecto de la tesis sobre la relación entre el derecho y la moral que ella acepta. Esta tesis se refiere a la determinación de parte de las condiciones que deben cumplirse para avalar una proposición jurídica. Más precisamente, en el razonamiento jurídico dirigido a establecer la norma a aplicar en el caso, las distintas versiones de las tesis de la conexión y de la separabilidad establecen las condiciones bajo las cuales resulta relevante la existencia de una norma moral $\mathrm{M}$ contraria a una norma supuestamente jurídica $\mathrm{N}$ para establecer su legalidad. ${ }^{46}$

Esto no es más que la reformulación del problema de la traducción. De hecho, los desacuerdos entre los partidarios de las distintas versiones de las tesis de la conexión y de la separabilidad son desacuerdos teóricos que, al mismo tiempo, se refieren a proposiciones interdisciplinarias y son, por tanto, desacuerdos teóricos interdisciplinarios. Para apreciar esto es adecuado descomponer la afirmación A sobre la relación entre $\mathrm{M}$ y $\mathrm{N}$ en tres afirmaciones más simples:

A1. "La contraparte sostiene que $\mathrm{N}$ es la norma jurídica a aplicar en el presente caso";

A2. "Existe una norma moral M aplicable al presente caso";

A3. "M va en contra de N".

Comencemos por las tesis extremas, C1 y S3. Según la tesis iusnaturalista fuerte $\mathrm{C} 1$, si el juez comparte $\mathrm{A} 1-\mathrm{A} 3$, entonces $\mathrm{N}$ no es una norma jurídica. Por el contrario, de acuerdo con la tesis positivista excluyente fuerte S3, que el juez comparta A1-A3 sería irrelevante ya que A3 es jurídicamente irrelevante.

46 Esto es una simplificación porque, por ejemplo, sobre la base de A, podría asumirse que $\mathrm{N}$ es una disposición legal pero que es inaplicable o no obligatoria. 
En el caso de las tesis intermedias, sin embargo, la afirmación del abogado es incompleta y puede ser considerada relevante por el juez solo si se cumplen condiciones más específicas. De acuerdo con la tesis iusnaturalista débil C2, el abogado debe apoyar algo más preciso que $\mathrm{A}$ y en particular que A3, a saber, A3": "M es intolerablemente contraria a N". La tesis del neoconstitucionalismo $\mathrm{C} 3$, en cambio, requiere una reformulación de A2, porque no es suficiente que exista la norma moral $\mathrm{M}$ para que sea relevante, sino que debe ser parte del derecho constitucional para determinar su relevancia y, por lo tanto, su aplicabilidad. Esto requiere una formulación diferente de A2, como A2': "Existe una regla moral $\mathrm{M}$ aplicable al presente caso en razón del derecho constitucional". La tesis positivista incluyente $\mathrm{S} 1$, al igual que el neoconstitucionalismo, modifica A2, pero dando más atención a los criterios de aval (o validez) establecidos por la regla de reconocimiento y al hecho de que ello implica la incorporación de una norma moral al derecho; A2": "Existe una norma moral M aplicable al caso en cuestión, ya que está incorporada al derecho". Finalmente, también para la tesis positivista excluyente débil S2, A2 debe modificarse. Esta vez, sin embargo, la modificación no determina la incorporación de la norma moral, sino solo su aplicabilidad; A3': "Existe una norma moral M aplicable al presente caso en la medida en que el derecho la hace aplicable".

El análisis anterior muestra cómo las diferentes tesis sobre la conexión y sobre la separabilidad del derecho y la moral representan diferentes criterios para determinar si la moral es relevante en la argumentación jurídica, y en caso afirmativo, bajo qué condiciones. Esto significa que estas tesis representan diferentes respuestas al problema de la traducción desde la moral al derecho. De hecho, estas tesis son criterios para considerar si una proposición moral sobre el derecho es relevante o no en la argumentación jurídica. En otras palabras, todas tienen como objetivo establecer si una proposición moral sobre el derecho es una proposición puramente interdisciplinaria o si puede llevar a un desacuerdo teórico interdisciplinario.

\section{CONCLUSIÓN}

En este ensayo, abordé un problema central a la relación entre las prácticas jurídicas y económicas, que luego mostré que es similar a un problema central de la teoría jurídica. Más precisamente, el problema de la traducción de una proposición desde la economía al derecho es similar al muy discutido problema de la relación entre la moral y el derecho. Los desacuerdos sobre las condiciones de relevancia de las proposiciones interdisciplinarias son de hecho, en ambos casos, desacuerdos teóricos interdisciplinarios. 
En el curso del análisis, sostuve que el problema de la traducción exige que la proposición económica (o moral) sea hecha parte de la justificación externa de una norma jurídica, es decir, transformar la proposición interdisciplinaria en un argumento jurídico. Las conclusiones sobre el problema de la traducción permiten determinar si un desacuerdo interdisciplinario en sentido estricto es, o no, también un desacuerdo teórico, dando lugar a un desacuerdo teórico interdisciplinario.

Desde una perspectiva más general, un aspecto destacable del análisis ofrecido en este ensayo es haber vinculado temas típicos de la filosofía del derecho y la teoría jurídica con un aspecto central de la relación entre el derecho y la economía. Por esta razón, me parece razonable sugerir a los filósofos y teóricos del derecho que deberían estar más interesados en la relación entre el derecho y la economía.

Sin embargo, una clara limitación del análisis es que está lejos de dar cuenta de las formas en que se puede abordar el problema de la traducción interdisciplinaria. Sin embargo, creo que este es un aspecto del problema de la traducción que es contingente $y$, por lo tanto, requiere un análisis que no se puede proporcionar aquí.

En cualquier caso, las consideraciones anteriores han resaltado dos aspectos del problema de la traducción que ciertamente pueden representar puntos de partida importantes para investigaciones más profundas. Se trata, por un lado, de la posibilidad de "traducciones implícitas". Se ha afirmado que este fenómeno es relevante también para la elaboración de autores como Hart y Alexy. Por otro lado, el análisis de la investigación de Habib ha mostrado que la traducción puede llevarse a cabo a nivel conceptual y, por lo tanto, no solo a través de alguna forma de argumento consecuencialista, como sugiere Patterson. En este sentido, la crítica a las reflexiones de Papayannis ha mostrado que es necesario ser cuidadoso al realizar actividades de traducción porque a menudo los economistas determinan los términos que utilizan dentro de análisis funcionales que bien pueden asumir una función respecto de una práctica regulada distinta de la que el derecho asigna a esa práctica. Esto requiere precaución. Es importante asegurarse de que las funciones que entran en conflicto con las asignadas por el derecho no acaben condicionando el contenido de este último debido a un error de traducción, especialmente si es implícita. Debido a la tendencia de los economistas a ser reacios a tener en cuenta el punto de vista de los juristas, parece plausible esperar que la terapia lingüística identificada en este ensayo caiga, en la práctica, sobre los hombros de los juristas. Así, entre los juristas, los filósofos y teóricos del derecho - especialmente los analíticos - están en una posición privilegiada para contribuir a un mejor diálogo entre el derecho y la economía. 


\section{BIBLIOGRAFÍA CITADA}

Alexy, R. (2002). A Theory of constitutional rights (traducción de J. Rivers, Oxford University Press).

Almeida, S.R. et al. (2016). Behavioural insights applied to policy: Overview across 32 European countries (European Union).

BARberis, M. (2011). “Diritto e morale: la discussione odierna”, Revus, Vol. 16 , pp. 55-93.

Calabresi, G. (2016). The Future of Law and Economics: Essays in Reform and Recollection (Yale University Press).

Canale, D. y Tuzet, G. (2007a). "On legal Inferentialism. Toward a Pragmatics of semantic Content in legal Interpretation?", Ratio Juris, Vol. 20, pp. 32-44.

Canale, D. y Tuzet, G. (2007b). “Judicial Scorekeeping”, en mismos autores, The Rules of Inference (Egea), pp. 73-89.

Canale, D. (2013). "Il ragionamento giuridico", en G. Pino, A. Schiavello, V. VILLA (eds.), Filosofia del diritto (Giappichelli), pp. 316-351.

Chiasssoni, P. (2016). "La terra promessa del professor Calabresi”, Contratto e impresa, Vol. 32, pp. 608-615.

Craswell, R. (1993). "Default rules, efficiency, and prudence", Southern California Interdisciplinary Law Journal, Vol. 3, pp.289-302.

CSERes, K.J. (2005). Competition law and consumer protection (Kluwer Law International).

CSERne, P. (2011). “Consequence-based arguments in legal reasoning”, en K. MaTHIS (ed.), Efficiency, sustainability and justice for future generations (Springer), pp. 31-54. 
Dworkin, R. (1986) Law's Empire (Fontana).

ESPOSITO, F. y GRUNDMANN, S. (2017). "'Investor-consumer or overall welfare: Searching for the paradigm of recent reforms in financial services contracts", Working papers of the Law Department of the EUI, 5, 2017 (https://ssrn.com/abstract=2925794)

Esposito, F. AlmeidA, L. de. (2018). "A shocking truth for Law and Economics: The internal market for electricity explained with consumer welfare", en K. Mathis and M. Huber (eds.), Energy law and economics in Europe (Springer).

Esposito, F. (2017a). "How the Behavioural Turn in Law and Economics vindicates the New Haven School”, Oeconomia, Vol. 7(3), pp. 375-406.

EsPosito, F. (2017b). "The dismal reality: Behavioural analysis and consumer policy", Journal of consumer policy, Vol. 2, 2017, pp. 193-216.

EsPosito, F. (2018) "Economic concepts in the analysis of proportionality reasoning between similarity and identity", Analisi e Diritto, 1/2018, pp. 185-210.

Esposito, F. (2019) "On the fitness between Law and Economics-Or Sunstein between Posner and Calabresi", Global Jurist, Vol. 19(3), pp. 1-11.

HaAck, S. (2015). Legalizzare l'epistemologia. Prova, probabilità e causalità nel diritto, (edición de G. Tuzet; traducción de F. Esposito, Università Bocconi Editore).

HaвIB, J.I. (2016). The methodology of private Law: Oron how to make private law recover its lost character (European University Institute, Florence).

Hart, H.L.A. (1994) The concept of law (2a ed., Oxford Clarendon Press).

HiLdeBRAND, H. (1998). The Role of economic analysis in EU competition law: the European school, Kluwer Law International, Alphen aan den Rijn 2016 (ed. or. 1998).

Hildebrand, H. (2005). "Interpretation in law", San Diego Law Review, Vol. 42, pp. 685-710.

Koczogh, H. (2013). "Scrutinizing the Concept of (verbal) Disagreement", Argumentum, Vol. 9, pp. 211-222. 
LeITER, B. (2009). "Explaining theoretical disagreement", University of Chicago Law Review, Vol. 76, pp. 1215-1250.

Lepenies, R. y Malecka, M. (2015). "Behavioural Sciences in Law and Policy: a Case of scientific Imperialism?”, en MALECKA, Magdalena y LePENIES, Robert, Behavioural Sciences in Law and Policy: A Case of Scientific Imperialism?, EUI Department of Law Research Paper No. MWP 2015/13, disponible en: https://ssrn.com/abstract $=2631760$

Lowe, P. (2007). "Consumer welfare and efficiency. New guiding principles of competitiveness policy?", discurso en la 13th International conference on competition and $14^{\text {th }}$ European Competition day, 27 de marzo de 2007, disponible en http://ec.europa.eu/ competition/speeches/text/sp2007_02_en.pdf

MäKI, U. (2013) "Scientific imperialism: Difficulties in definition, identification, and assessment", International studies in the philosophy of science, Vol. 7, pp. 325-339

PaPayanNis, D.M. (2013a) Spiegazione funzionale e analisi concettuale. Sull'impatto dei modelli economici sullo studio della pratica giuridica, in "Ars interpretandi", 4, 2013, pp. 69-110

PAPAYANNIS, D.M. (2013b) "Legality: between purposes and functions", en D. Canale y G. Tuzet (eds.), The planning theory of law (Springer), pp. $89-128$.

PATterson, D. (1993). "The pseudo-debate over default rules in contract law", Southern California Interdisciplinary Law Journal, Vol. 3, pp. 235-288.

Patterson, D. (2005). "Interpretation in Law”, San Diego Law Review, Vol. 42, pp. 685-710.

PINO, G. (2015). "La norma di riconoscimento come ideologia delle fonti", Analisi e diritto, 2015, pp. 183-201.

RAmírez LudeÑA, L. (2016). “Legal disagreements. A pluralist reply to Dworkin's challenge”, Revus, Vol. XXVIII, pp. 11-32.

Ratti, G.B. y Ferrer, J. (2013). "Theoretical disagreements: A restatement of legal positivism", en D. CANale y G. Tuzet (eds.), The planning theory of law (Springer), pp. 169-186. 
Ratti, G.B. y Dolcetti, A. (2013). "Legal disagreements and the dual nature of law", en S. SciarafFa y W. Waluchow (eds.), Philosophical foundations of the nature of law (Oxford University Press).

SchweIzer, U. (2009). "Contract remedies from the incentive perspective", en G. WAGNER (ed.), The common frame of reference: A view from Law \& Economics (European Law Publishers), pp. 1-18.

SHAPIRO, S.J. (2007). “The Hart-Dworkin debate: A short guide for the perplexed", en A. RiPSTEIN (ed.), Ronald Dworkin (Cambridge University Press), pp. 22-55.

SHAPIRO, S.J. (2009). "What is the rule of recognition (and does it exist)?", en M. D. Alder y K. Himma (eds.), The rule of recognition and the U.S. constitution (Oxford University Press), pp. 235-268.

SHAPIRO, S.J. (2011). Legality (Harvard University Press).

Wròbleski, J. (1992). The judicial Application of Law (edición de Z. Bańkowski y N. MacCormick, Kluwer Academic Publishers). 